\title{
Removing Camera Shake from a Single Photograph
}

\author{
Rob Fergus ${ }^{1} \quad$ Barun Singh $^{1} \quad$ Aaron Hertzmann $^{2} \quad$ Sam T. Roweis $^{2} \quad$ William T. Freeman ${ }^{1}$ \\ ${ }^{1}$ MIT CSAIL $\quad{ }^{2}$ University of Toronto
}
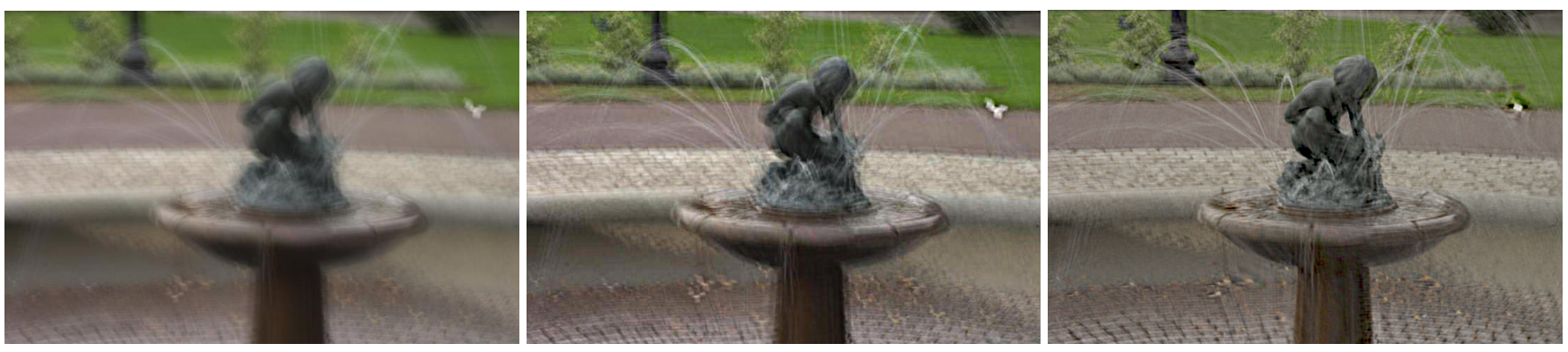

Figure 1: Left: An image spoiled by camera shake. Middle: result from Photoshop "unsharp mask". Right: result from our algorithm.

\begin{abstract}
Camera shake during exposure leads to objectionable image blur and ruins many photographs. Conventional blind deconvolution methods typically assume frequency-domain constraints on images, or overly simplified parametric forms for the motion path during camera shake. Real camera motions can follow convoluted paths, and a spatial domain prior can better maintain visually salient image characteristics. We introduce a method to remove the effects of camera shake from seriously blurred images. The method assumes a uniform camera blur over the image and negligible in-plane camera rotation. In order to estimate the blur from the camera shake, the user must specify an image region without saturation effects. We show results for a variety of digital photographs taken from personal photo collections.
\end{abstract}

CR Categories: I.4.3 [Image Processing and Computer Vision]: Enhancement, G.3 [Artificial Intelligence]: Learning

Keywords: camera shake, blind image deconvolution, variational learning, natural image statistics

\section{Introduction}

Camera shake, in which an unsteady camera causes blurry photographs, is a chronic problem for photographers. The explosion of consumer digital photography has made camera shake very prominent, particularly with the popularity of small, high-resolution cameras whose light weight can make them difficult to hold sufficiently steady. Many photographs capture ephemeral moments that cannot be recaptured under controlled conditions or repeated with different camera settings - if camera shake occurs in the image for any reason, then that moment is "lost".

Shake can be mitigated by using faster exposures, but that can lead to other problems such as sensor noise or a smaller-than-desired depth-of-field. A tripod, or other specialized hardware, can eliminate camera shake, but these are bulky and most consumer photographs are taken with a conventional, handheld camera. Users may avoid the use of flash due to the unnatural tonescales that result. In our experience, many of the otherwise favorite photographs of amateur photographers are spoiled by camera shake. A method to remove that motion blur from a captured photograph would be an important asset for digital photography.

Camera shake can be modeled as a blur kernel, describing the camera motion during exposure, convolved with the image intensities. Removing the unknown camera shake is thus a form of blind image deconvolution, which is a problem with a long history in the image and signal processing literature. In the most basic formulation, the problem is underconstrained: there are simply more unknowns (the original image and the blur kernel) than measurements (the observed image). Hence, all practical solutions must make strong prior assumptions about the blur kernel, about the image to be recovered, or both. Traditional signal processing formulations of the problem usually make only very general assumptions in the form of frequency-domain power laws; the resulting algorithms can typically handle only very small blurs and not the complicated blur kernels often associated with camera shake. Furthermore, algorithms exploiting image priors specified in the frequency domain may not preserve important spatial-domain structures such as edges.

This paper introduces a new technique for removing the effects of unknown camera shake from an image. This advance results from two key improvements over previous work. First, we exploit recent research in natural image statistics, which shows that photographs of natural scenes typically obey very specific distributions of image gradients. Second, we build on work by Miskin and MacKay [2000], adopting a Bayesian approach that takes into account uncertainties in the unknowns, allowing us to find the blur kernel implied by a distribution of probable images. Given this kernel, the image is then reconstructed using a standard deconvolution algorithm, although we believe there is room for substantial improvement in this reconstruction phase.

We assume that all image blur can be described as a single convolution; i.e., there is no significant parallax, any image-plane rotation of the camera is small, and no parts of the scene are moving relative to one another during the exposure. Our approach currently requires a small amount of user input.

Our reconstructions do contain artifacts, particularly when the 
above assumptions are violated; however, they may be acceptable to consumers in some cases, and a professional designer could touchup the results. In contrast, the original images are typically unusable, beyond touching-up - in effect our method can help "rescue" shots that would have otherwise been completely lost.

\section{Related Work}

The task of deblurring an image is image deconvolution; if the blur kernel is not known, then the problem is said to be "blind". For a survey on the extensive literature in this area, see [Kundur and Hatzinakos 1996]. Existing blind deconvolution methods typically assume that the blur kernel has a simple parametric form, such as a Gaussian or low-frequency Fourier components. However, as illustrated by our examples, the blur kernels induced during camera shake do not have simple forms, and often contain very sharp edges. Similar low-frequency assumptions are typically made for the input image, e.g., applying a quadratic regularization. Such assumptions can prevent high frequencies (such as edges) from appearing in the reconstruction. Caron et al. [2002] assume a power-law distribution on the image frequencies; power-laws are a simple form of natural image statistics that do not preserve local structure. Some methods [Jalobeanu et al. 2002; Neelamani et al. 2004] combine power-laws with wavelet domain constraints but do not work for the complex blur kernels in our examples.

Deconvolution methods have been developed for astronomical images [Gull 1998; Richardson 1972; Tsumuraya et al. 1994; Zarowin 1994], which have statistics quite different from the natural scenes we address in this paper. Performing blind deconvolution in this domain is usually straightforward, as the blurry image of an isolated star reveals the point-spread-function.

Another approach is to assume that there are multiple images available of the same scene [Bascle et al. 1996; Rav-Acha and Peleg 2005]. Hardware approaches include: optically stabilized lenses [Canon Inc. 2006], specially designed CMOS sensors [Liu and Gamal 2001], and hybrid imaging systems [Ben-Ezra and Nayar 2004]. Since we would like our method to work with existing cameras and imagery and to work for as many situations as possible, we do not assume that any such hardware or extra imagery is available.

Recent work in computer vision has shown the usefulness of heavytailed natural image priors in a variety of applications, including denoising [Roth and Black 2005], superresolution [Tappen et al. 2003], intrinsic images [Weiss 2001], video matting [Apostoloff and Fitzgibbon 2005], inpainting [Levin et al. 2003], and separating reflections [Levin and Weiss 2004]. Each of these methods is effectively "non-blind", in that the image formation process (e.g., the blur kernel in superresolution) is assumed to be known in advance.

Miskin and MacKay [2000] perform blind deconvolution on line art images using a prior on raw pixel intensities. Results are shown for small amounts of synthesized image blur. We apply a similar variational scheme for natural images using image gradients in place of intensities and augment the algorithm to achieve results for photographic images with significant blur.

\section{Image model}

Our algorithm takes as input a blurred input image $\mathbf{B}$, which is assumed to have been generated by convolution of a blur kernel $\mathbf{K}$ with a latent image $\mathbf{L}$ plus noise:

$$
\mathbf{B}=\mathbf{K} \otimes \mathbf{L}+\mathbf{N}
$$

where $\otimes$ denotes discrete image convolution (with non-periodic boundary conditions), and $\mathbf{N}$ denotes sensor noise at each pixel. We assume that the pixel values of the image are linearly related to
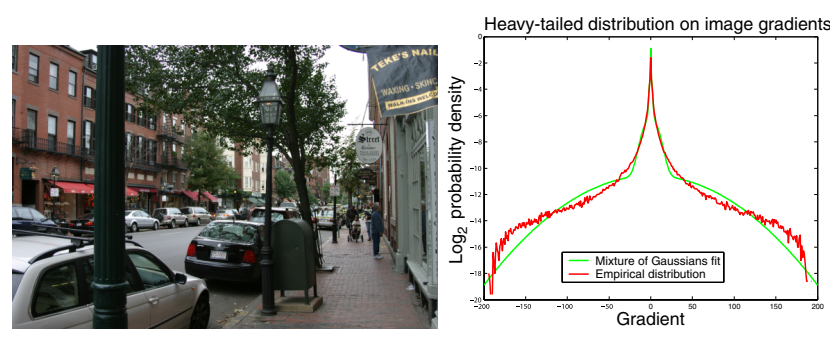

Figure 2: Left: A natural scene. Right: The distribution of gradient magnitudes within the scene are shown in red. The $y$-axis has a logarithmic scale to show the heavy tails of the distribution. The mixture of Gaussians approximation used in our experiments is shown in green.

the sensor irradiance. The latent image $\mathbf{L}$ represents the image we would have captured if the camera had remained perfectly still; our goal is to recover $\mathbf{L}$ from $\mathbf{B}$ without specific knowledge of $\mathbf{K}$.

In order to estimate the latent image from such limited measurements, it is essential to have some notion of which images are $a$ priori more likely. Fortunately, recent research in natural image statistics have shown that, although images of real-world scenes vary greatly in their absolute color distributions, they obey heavytailed distributions in their gradients [Field 1994]: the distribution of gradients has most of its mass on small values but gives significantly more probability to large values than a Gaussian distribution. This corresponds to the intuition that images often contain large sections of constant intensity or gentle intensity gradient interrupted by occasional large changes at edges or occlusion boundaries. For example, Figure 2 shows a natural image and a histogram of its gradient magnitudes. The distribution shows that the image contains primarily small or zero gradients, but a few gradients have large magnitudes. Recent image processing methods based on heavy-tailed distributions give state-of-the-art results in image denoising [Roth and Black 2005; Simoncelli 2005] and superresolution [Tappen et al. 2003]. In contrast, methods based on Gaussian prior distributions (including methods that use quadratic regularizers) produce overly smooth images.

We represent the distribution over gradient magnitudes with a zeromean mixture-of-Gaussians model, as illustrated in Figure 2. This representation was chosen because it can provide a good approximation to the empirical distribution, while allowing a tractable estimation procedure for our algorithm.

\section{Algorithm}

There are two main steps to our approach. First, the blur kernel is estimated from the input image. The estimation process is performed in a coarse-to-fine fashion in order to avoid local minima. Second, using the estimated kernel, we apply a standard deconvolution algorithm to estimate the latent (unblurred) image.

The user supplies four inputs to the algorithm: the blurred image $\mathbf{B}$, a rectangular patch within the blurred image, an upper bound on the size of the blur kernel (in pixels), and an initial guess as to orientation of the blur kernel (horizontal or vertical). Details of how to specify these parameters are given in Section 4.1.2.

Additionally, we require input image $\mathbf{B}$ to have been converted to a linear color space before processing. In our experiments, we applied inverse gamma-correction ${ }^{1}$ with $\gamma=2.2$. In order to estimate the expected blur kernel, we combine all the color channels of the original image within the user specified patch to produce a grayscale blurred patch $\mathbf{P}$.

\footnotetext{
${ }^{1}$ Pixel value $=(\text { CCD sensor value })^{1 / \gamma}$
} 


\subsection{Estimating the blur kernel}

Given the grayscale blurred patch $\mathbf{P}$, we estimate $\mathbf{K}$ and the latent patch image $\mathbf{L}_{p}$ by finding the values with highest probability, guided by a prior on the statistics of $\mathbf{L}$. Since these statistics are based on the image gradients rather than the intensities, we perform the optimization in the gradient domain, using $\nabla \mathbf{L}_{p}$ and $\nabla \mathbf{P}$, the gradients of $\mathbf{L}_{p}$ and $\mathbf{P}$. Because convolution is a linear operation, the patch gradients $\nabla \mathbf{P}$ should be equal to the convolution of the latent gradients and the kernel: $\nabla \mathbf{P}=\nabla \mathbf{L}_{p} \otimes \mathbf{K}$, plus noise. We assume that this noise is Gaussian with variance $\sigma^{2}$.

As discussed in the previous section, the prior $p\left(\nabla \mathbf{L}_{p}\right)$ on the latent image gradients is a mixture of $C$ zero-mean Gaussians (with variance $v_{c}$ and weight $\pi_{c}$ for the $c$-th Gaussian). We use a sparsity prior $p(\mathbf{K})$ for the kernel that encourages zero values in the kernel, and requires all entries to be positive. Specifically, the prior on kernel values is a mixture of $D$ exponential distributions (with scale factors $\lambda_{d}$ and weights $\pi_{d}$ for the $d$-th component).

Given the measured image gradients $\nabla \mathbf{P}$, we can write the posterior distribution over the unknowns with Bayes' Rule:

$$
\begin{aligned}
p\left(\mathbf{K}, \nabla \mathbf{L}_{p} \mid \nabla \mathbf{P}\right) \propto & p\left(\nabla \mathbf{P} \mid \mathbf{K}, \nabla \mathbf{L}_{p}\right) p\left(\nabla \mathbf{L}_{p}\right) p(\mathbf{K}) \\
= & \prod_{i} \mathrm{~N}\left(\nabla \mathbf{P}(i) \mid\left(\mathbf{K} \otimes \nabla \mathbf{L}_{p}(i)\right), \sigma^{2}\right) \\
& \prod_{i} \sum_{c=1}^{C} \pi_{c} \mathrm{~N}\left(\nabla \mathbf{L}_{p}(i) \mid 0, v_{c}\right) \prod_{j} \sum_{d=1}^{D} \pi_{d} \mathrm{E}\left(\mathbf{K}_{j} \mid \lambda_{d}\right)
\end{aligned}
$$

where $i$ indexes over image pixels and $j$ indexes over blur kernel elements. $\mathrm{N}$ and $\mathrm{E}$ denote Gaussian and Exponential distributions respectively. For tractability, we assume that the gradients in $\nabla \mathbf{P}$ are independent of each other, as are the elements in $\nabla \mathbf{L}_{p}$ and $\mathbf{K}$.

A straightforward approach to deconvolution is to solve for the maximum a-posteriori (MAP) solution, which finds the kernel $\mathbf{K}$ and latent image gradients $\nabla \mathbf{L}$ that maximizes $p\left(\mathbf{K}, \nabla \mathbf{L}_{p} \mid \nabla \mathbf{P}\right)$. This is equivalent to solving a regularized-least squares problem that attempts to fit the data while also minimizing small gradients. We tried this (using conjugate gradient search) but found that the algorithm failed. One interpretation is that the MAP objective function attempts to minimize all gradients (even large ones), whereas we expect natural images to have some large gradients. Consequently, the algorithm yields a two-tone image, since virtually all the gradients are zero. If we reduce the noise variance (thus increasing the weight on the data-fitting term), then the algorithm yields a deltafunction for $\mathbf{K}$, which exactly fits the blurred image, but without any deblurring. Additionally, we find the MAP objective function to be very susceptible to poor local minima.

Instead, our approach is to approximate the full posterior distribution $p\left(\mathbf{K}, \nabla \mathbf{L}_{p} \mid \nabla \mathbf{P}\right)$, and then compute the kernel $\mathbf{K}$ with maximum marginal probability. This method selects a kernel that is most likely with respect to the distribution of possible latent images, thus avoiding the overfitting that can occur when selecting a single "best" estimate of the image.

In order to compute this approximation efficiently, we adopt a variational Bayesian approach [Jordan et al. 1999] which computes a distribution $q\left(\mathbf{K}, \nabla \mathbf{L}_{p}\right)$ that approximates the posterior $p\left(\mathbf{K}, \nabla \mathbf{L}_{p} \mid \nabla \mathbf{P}\right)$. In particular, our approach is based on Miskin and MacKay's algorithm [2000] for blind deconvolution of cartoon images. A factored representation is used: $q\left(\mathbf{K}, \nabla \mathbf{L}_{p}\right)=q(\mathbf{K}) q\left(\nabla \mathbf{L}_{p}\right)$. For the latent image gradients, this approximation is a Gaussian density, while for the non-negative blur kernel elements, it is a rectified Gaussian. The distributions for each latent gradient and blur kernel element are represented by their mean and variance, stored in an array.
Following Miskin and MacKay [2000], we also treat the noise variance $\sigma^{2}$ as an unknown during the estimation process, thus freeing the user from tuning this parameter. This allows the noise variance to vary during estimation: the data-fitting constraint is loose early in the process, becoming tighter as better, low-noise solutions are found. We place a prior on $\sigma^{2}$, in the form of a Gamma distribution on the inverse variance, having hyper-parameters $a, b: p\left(\sigma^{2} \mid a, b\right)=$ $\Gamma\left(\sigma^{-2} \mid a, b\right)$. The variational posterior of $\sigma^{2}$ is $q\left(\sigma^{-2}\right)$, another Gamma distribution.

The variational algorithm minimizes a cost function representing the distance between the approximating distribution and the true posterior, measured as: $K L\left(q\left(\mathbf{K}, \nabla \mathbf{L}_{p}, \sigma^{-2}\right) \| p\left(\mathbf{K}, \nabla \mathbf{L}_{p} \mid \nabla \mathbf{P}\right)\right)$. The independence assumptions in the variational posterior allows the cost function $C_{K L}$ to be factored:

$$
<\log \frac{q\left(\nabla \mathbf{L}_{p}\right)}{p\left(\nabla \mathbf{L}_{p}\right)}>_{q\left(\nabla \mathbf{L}_{p}\right)}+<\log \frac{q(\mathbf{K})}{p(\mathbf{K})}>_{q(\mathbf{K})}+<\log \frac{q\left(\sigma^{-2}\right)}{p\left(\sigma^{2}\right)}>_{q\left(\sigma^{-2}\right)}
$$

where $\langle\cdot\rangle_{q(\theta)}$ denotes the expectation with respect to $q(\theta)^{2}$. For brevity, the dependence on $\nabla \mathbf{P}$ is omitted from this equation.

The cost function is then minimized as follows. The means of the distributions $q(\mathbf{K})$ and $q\left(\nabla \mathbf{L}_{p}\right)$ are set to the initial values of $\mathbf{K}$ and $\nabla \mathbf{L}_{p}$ and the variance of the distributions set high, reflecting the lack of certainty in the initial estimate. The parameters of the distributions are then updated alternately by coordinate descent; one is updated by marginalizing out over the other whilst incorporating the model priors. Updates are performed by computing closedform optimal parameter updates, and performing line-search in the direction of these updated values (see Appendix A for details). The updates are repeated until the change in $C_{K L}$ becomes negligible. The mean of the marginal distribution $\langle\mathbf{K}\rangle_{q(\mathbf{K})}$ is then taken as the final value for $\mathbf{K}$. Our implementation adapts the source code provided online by Miskin and MacKay [2000a].

In the formulation outlined above, we have neglected the possibility of saturated pixels in the image, an awkward non-linearity which violates our model. Since dealing with them explicitly is complicated, we prefer to simply mask out saturated regions of the image during the inference procedure, so that no use is made of them.

For the variational framework, $C=D=4$ components were used in the priors on $\mathbf{K}$ and $\nabla \mathbf{L}_{p}$. The parameters of the prior on the latent image gradients $\pi_{c}, v_{c}$ were estimated from a single street scene image, shown in Figure 2, using EM. Since the image statistics vary across scale, each scale level had its own set of prior parameters. This prior was used for all experiments. The parameters for the prior on the blur kernel elements were estimated from a small set of low-noise kernels inferred from real images.

\subsubsection{Multi-scale approach}

The algorithm described in the previous section is subject to local minima, particularly for large blur kernels. Hence, we perform estimation by varying image resolution in a coarse-to-fine manner. At the coarsest level, $\mathbf{K}$ is a $3 \times 3 \mathrm{kernel}$. To ensure a correct start to the algorithm, we manually specify the initial $3 \times 3$ blur kernel to one of two simple patterns (see Section 4.1.2). The initial estimate for the latent gradient image is then produced by running the inference scheme, while holding $\mathbf{K}$ fixed.

We then work back up the pyramid running the inference at each level; the converged values of $\mathbf{K}$ and $\nabla \mathbf{L}_{p}$ being upsampled to act as an initialization for inference at the next scale up. At the finest scale, the inference converges to the full resolution kernel $\mathbf{K}$.

\footnotetext{
${ }^{2}$ For example, $\left\langle\sigma^{-2}\right\rangle_{q\left(\sigma^{-2}\right)}=\int_{\sigma^{-2}} \sigma^{-2} \Gamma\left(\sigma^{-2} \mid a, b\right)=b / a$.
} 


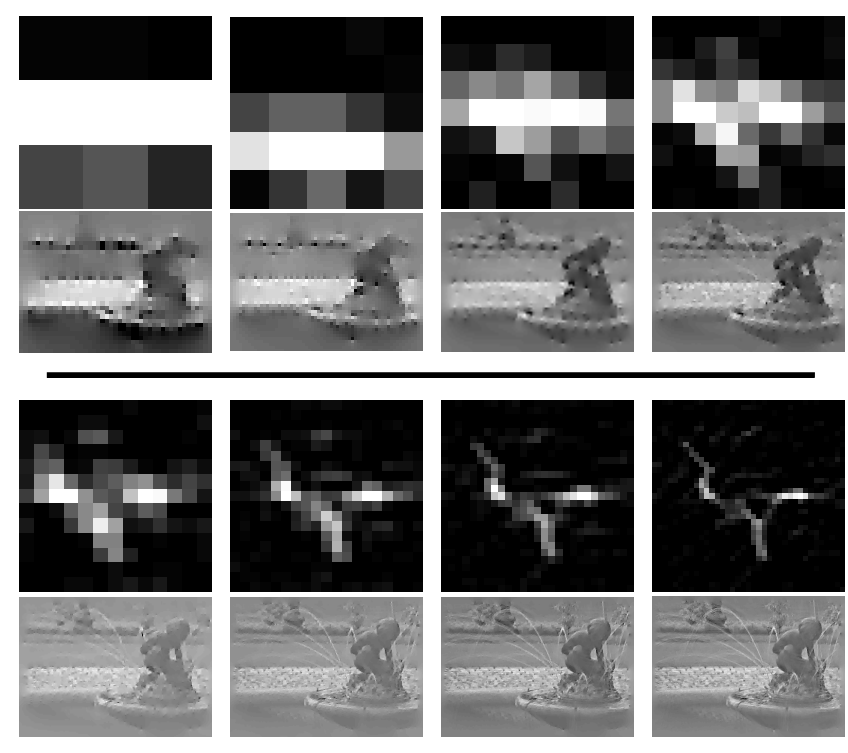

Figure 3: The multi-scale inference scheme operating on the fountain image in Figure 1. 1st \& 3rd rows: The estimated blur kernel at each scale level. $2 n d \& 4$ th rows: Estimated image patch at each scale. The intensity image was reconstructed from the gradients used in the inference using Poisson image reconstruction. The Poisson reconstructions are shown for reference only; the final reconstruction is found using the Richardson-Lucy algorithm with the final estimated blur kernel.

\subsubsection{User supervision}

Although it would seem more natural to run the multi-scale inference scheme using the full gradient image $\nabla \mathbf{L}$, in practice we found the algorithm performed better if a smaller patch, rich in edge structure, was manually selected. The manual selection allows the user to avoid large areas of saturation or uniformity, which can be disruptive or uninformative to the algorithm. Examples of user-selected patches are shown in Section 5. Additionally, the algorithm runs much faster on a small patch than on the entire image.

An additional parameter is that of the maximum size of the blur kernel. The size of the blur encountered in images varies widely, from a few pixels up to hundreds. Small blurs are hard to resolve if the algorithm is initialized with a very large kernel. Conversely, large blurs will be cropped if too small a kernel is used. Hence, for operation under all conditions, the approximate size of the kernel is a required input from the user. By examining any blur artifact in the image, the size of the kernel is easily deduced.

Finally, we also require the user to select between one of two initial estimates of the blur kernel: a horizontal line or a vertical line. Although the algorithm can often be initialized in either state and still produce the correct high resolution kernel, this ensures the algorithm starts searching in the correct direction. The appropriate initialization is easily determined by looking at any blur kernel artifact in the image.

\subsection{Image Reconstruction}

The multi-scale inference procedure outputs an estimate of the blur kernel $\mathbf{K}$, marginalized over all possible image reconstructions. To recover the deblurred image given this estimate of the kernel, we experimented with a variety of non-blind deconvolution methods, including those of Geman [1992], Neelamani [2004] and van Cittert [Zarowin 1994]. While many of these methods perform well in synthetic test examples, our real images exhibit a range of nonlinearities not present in synthetic cases, such as non-Gaussian noise, saturated pixels, residual non-linearities in tonescale and estimation errors in the kernel. Disappointingly, when run on our images, most methods produced unacceptable levels of artifacts.

We also used our variational inference scheme on the gradients of the whole image $\nabla \mathbf{B}$, while holding $\mathbf{K}$ fixed. The intensity image was then formed via Poisson image reconstruction [Weiss 2001]. Aside from being slow, the inability to model the non-linearities mentioned above resulted in reconstructions no better than other approaches.

As $\mathbf{L}$ typically is large, speed considerations make simple methods attractive. Consequently, we reconstruct the latent color image $\mathbf{L}$ with the Richardson-Lucy (RL) algorithm [Richardson 1972; Lucy 1974]. While the RL performed comparably to the other methods evaluated, it has the advantage of taking only a few minutes, even on large images (other, more complex methods, took hours or days). $\mathrm{RL}$ is a non-blind deconvolution algorithm that iteratively maximizes the likelihood function of a Poisson statistics image noise model. One benefit of this over more direct methods is that it gives only non-negative output values. We use Matlab's implementation of the algorithm to estimate $\mathbf{L}$, given $\mathbf{K}$, treating each color channel independently. We used $10 \mathrm{RL}$ iterations, although for large blur kernels, more may be needed. Before running RL, we clean up $\mathbf{K}$ by applying a dynamic threshold, based on the maximum intensity value within the kernel, which sets all elements below a certain value to zero, so reducing the kernel noise. The output of RL was then gamma-corrected using $\gamma=2.2$ and its intensity histogram matched to that of $\mathbf{B}$ (using Matlab's histeq function), resulting in L. See pseudo-code in Appendix A for details.

\section{Experiments}

We performed an experiment to check that blurry images are mainly due to camera translation as opposed to other motions, such as in-plane rotation. To this end, we asked 8 people to photograph a whiteboard ${ }^{3}$ which had small black dots placed in each corner whilst using a shutter speed of 1 second. Figure 4 shows dots extracted from a random sampling of images taken by different people. The dots in each corner reveal the blur kernel local to that portion of the image. The blur patterns are very similar, showing that our assumptions of spatially invariant blur with little in plane rotation are valid.

We apply our algorithm to a number of real images with varying degrees of blur and saturation. All the photos came from personal photo collections, with the exception of the fountain and cafe images which were taken with a high-end DSLR using long exposures ( $>1 / 2$ second). For each we show the blurry image, followed by the output of our algorithm along with the estimated kernel.

The running time of the algorithm is dependent on the size of the patch selected by the user. With the minimum practical size of $128 \times 128$ it currently takes 10 minutes in our Matlab implementation. For a patch of $N$ pixels, the run-time is $O(N \log N)$ owing to our use of FFT's to perform the convolution operations. Hence larger patches will still run in a reasonable time. Compiled and optimized versions of our algorithm could be expected to run considerably faster.

Small blurs. Figures 5 and 6 show two real images degraded by small blurs that are significantly sharpened by our algorithm. The

\footnotetext{
${ }^{3}$ Camera-to-whiteboard distance was $\approx 5 \mathrm{~m}$. Lens focal length was $50 \mathrm{~mm}$ mounted on a $0.6 \mathrm{x}$ DSLR sensor.
} 

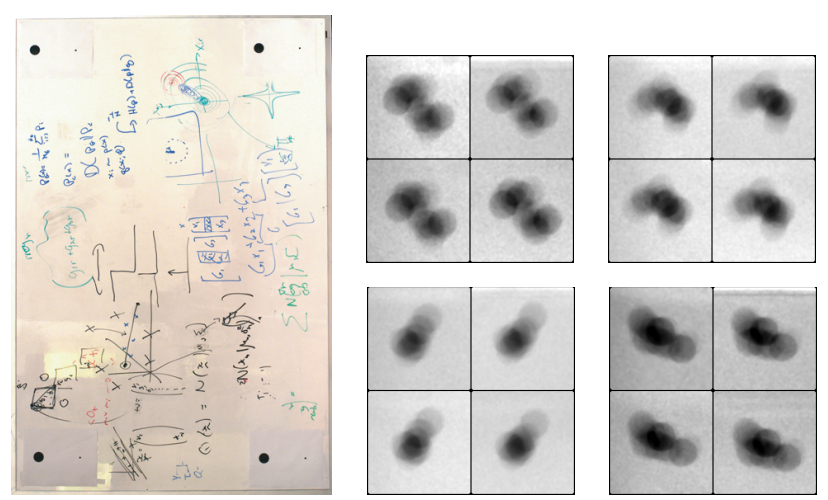

Figure 4: Left: The whiteboard test scene with dots in each corner. Right: Dots from the corners of images taken by different people. Within each image, the dot trajectories are very similar suggesting that image blur is well modeled as a spatially invariant convolution.

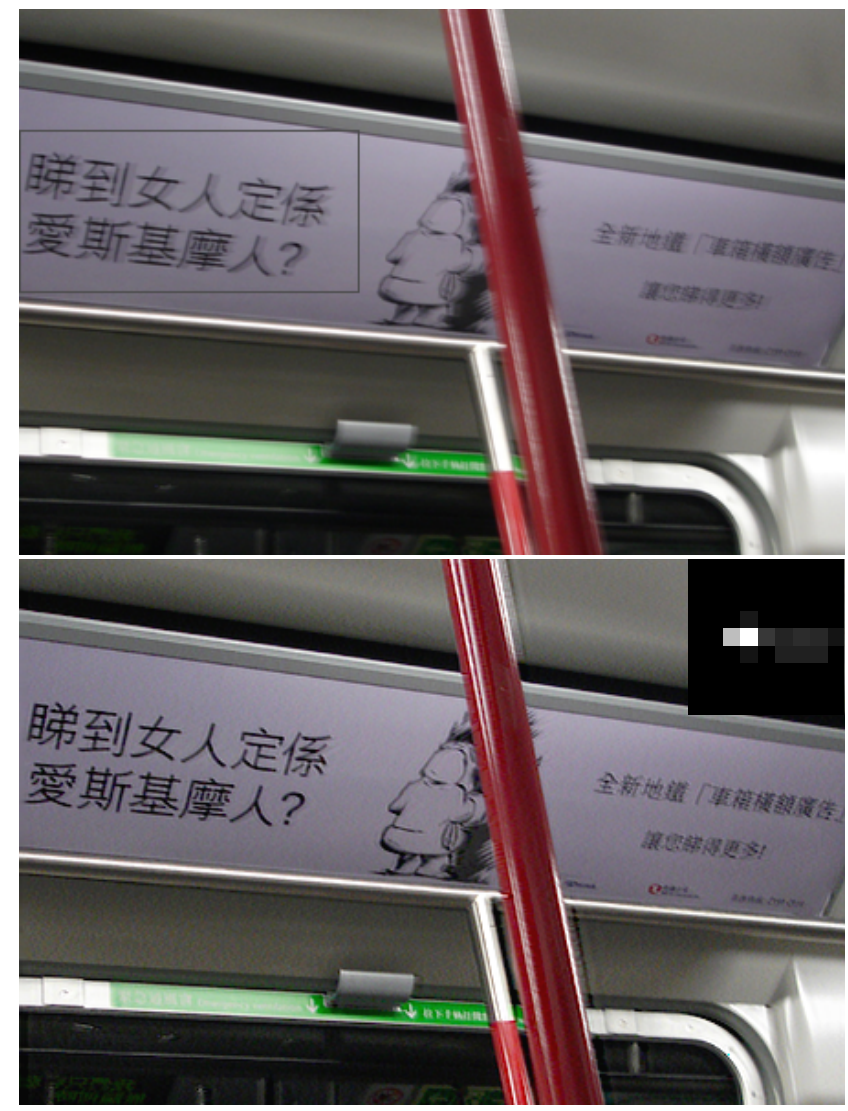

Figure 5: Top: A scene with a small blur. The patch selected by the user is indicated by the gray rectangle. Bottom: Output of our algorithm and the inferred blur kernel. Note the crisp text.

gray rectangles show the patch used to infer the blur kernel, chosen to have many image details but few saturated pixels. The inferred kernels are shown in the corner of the deblurred images.

Large blurs. Unlike existing blind deconvolution methods our algorithm can handle large, complex blurs. Figures 7 and 9 show our algorithm successfully inferring large blur kernels. Figure 1 shows an image with a complex tri-lobed blur, 30 pixels in size (shown in Figure 10), being deblurred.
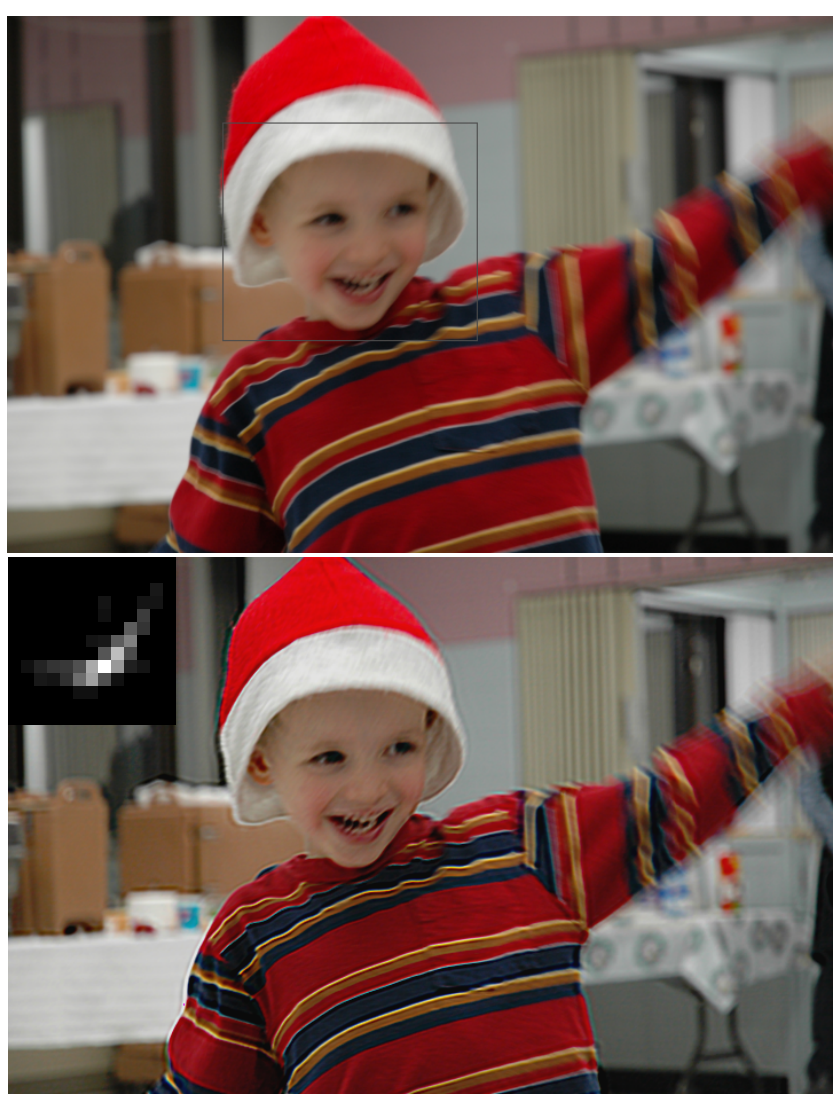

Figure 6: Top: A scene with complex motions. While the motion of the camera is small, the child is both translating and, in the case of the arm, rotating. Bottom: Output of our algorithm. The face and shirt are sharp but the arm remains blurred, its motion not modeled by our algorithm.

As demonstrated in Figure 8, the true blur kernel is occasionally revealed in the image by the trajectory of a point light source transformed by the blur. This gives us an opportunity to compare the inferred blur kernel with the true one. Figure 10 shows four such image structures, along with the inferred kernels from the respective images.

We also compared our algorithm against existing blind deconvolution algorithms, running Matlab's deconvblind routine, which provides implementations of the methods of Biggs and Andrews [1997] and Jansson [1997]. Based on the iterative Richardson-Lucy scheme, these methods also estimate the blur kernel; alternating between holding the blur constant and updating the image and viceversa. The results of this algorithm, applied to the fountain and cafe scenes are shown in Figure 11 and are poor compared to the output of our algorithm, shown in Figures 1 and 13.

Images with significant saturation. Figures 12 and 13 contain large areas where the true intensities are not observed, owing to the dynamic range limitations of the camera. The user-selected patch used for kernel analysis must avoid the large saturated regions. While the deblurred image does have some artifacts near saturated regions, the unsaturated regions can still be extracted. 


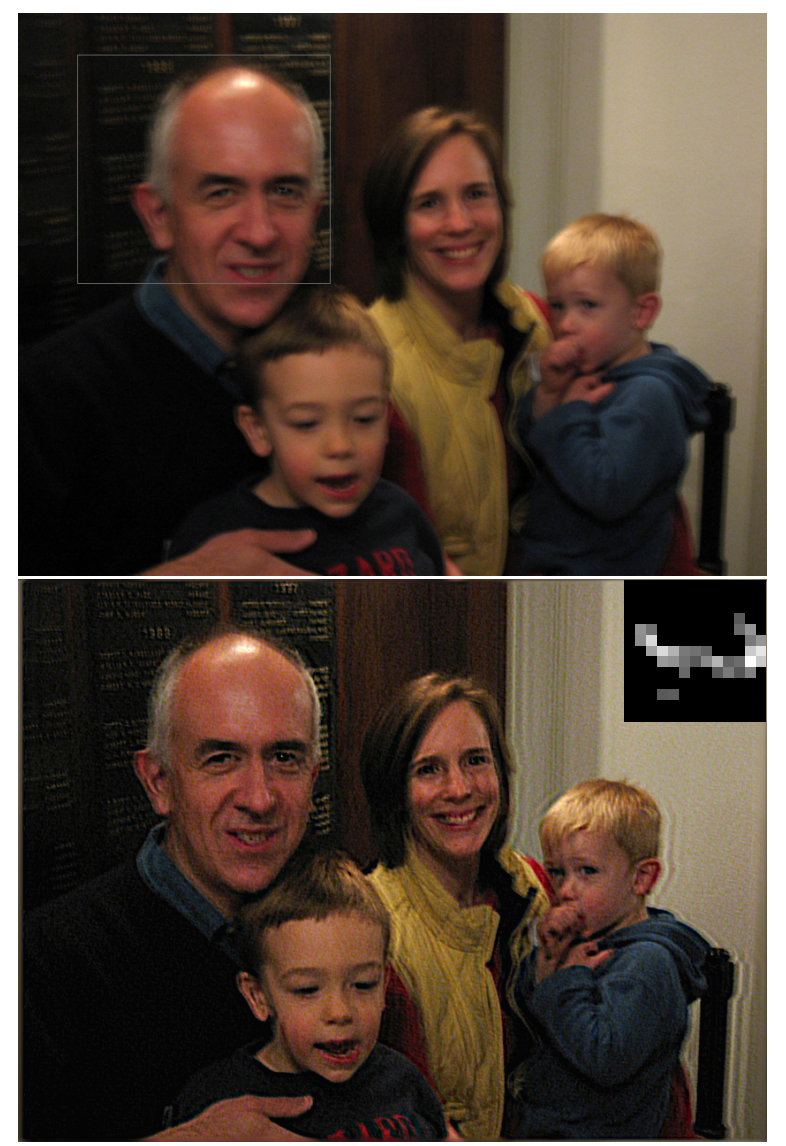

Figure 7: Top: A scene with a large blur. Bottom: Output of our algorithm. See Figure 8 for a closeup view.
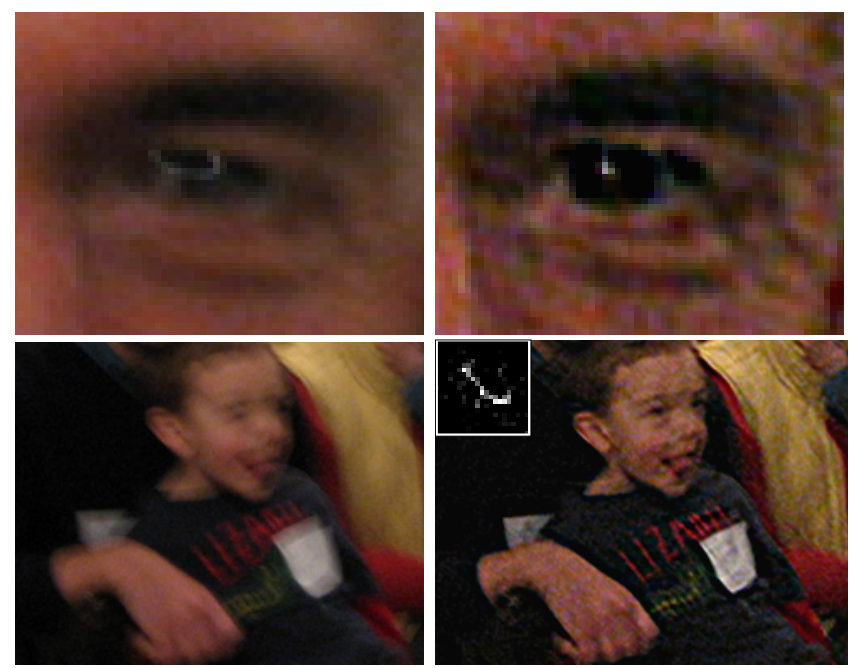

Figure 8: Top row: Closeup of the man's eye in Figure 7. The original image (on left) shows a specularity distorted by the camera motion. In the deblurred image (on right) the specularity is condensed to a point. The color noise artifacts due to low light exposure can be removed by median filtering the chrominance channels. Bottom row: Closeup of child from another image of the family (different from Figure 7). In the deblurred image, the text on his jersey is now legible.

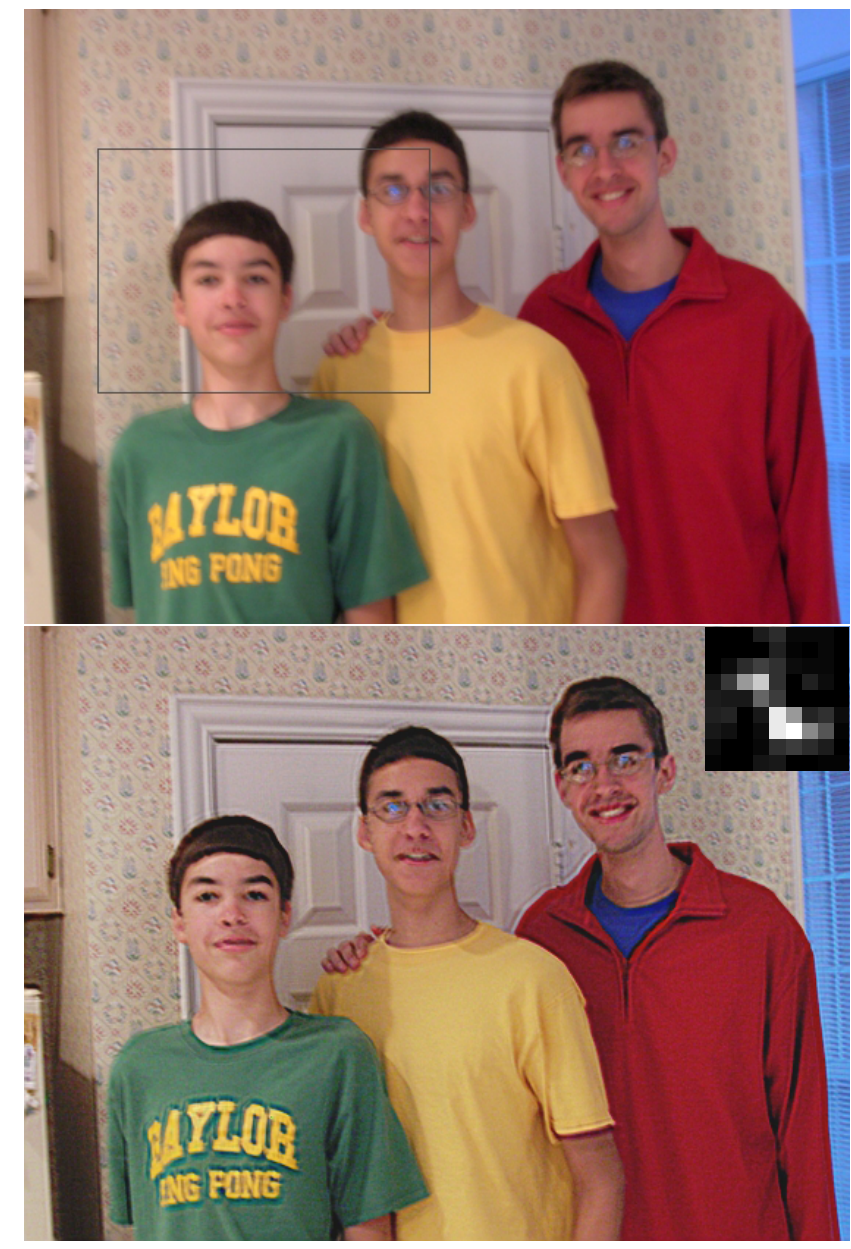

Figure 9: Top: A blurry photograph of three brothers. Bottom: Output of our algorithm. The fine detail of the wallpaper is now visible.

\section{Discussion}

We have introduced a method for removing camera shake effects from photographs. This problem appears highly underconstrained at first. However, we have shown that by applying natural image priors and advanced statistical techniques, plausible results can nonetheless be obtained. Such an approach may prove useful in other computational photography problems.

Most of our effort has focused on kernel estimation, and, visually, the kernels we estimate seem to match the image camera motion. The results of our method often contain artifacts; most prominently, ringing artifacts occur near saturated regions and regions of significant object motion. We suspect that these artifacts can be blamed primarily on the non-blind deconvolution step. We believe that there is significant room for improvement by applying modern statistical methods to the non-blind deconvolution problem.

There are a number of common photographic effects that we do not explicitly model, including saturation, object motion, and compression artifacts. Incorporating these factors into our model should improve robustness. Currently we assume images to have a linear tonescale, once the gamma correction has been removed. However, cameras typically have a slight sigmoidal shape to their tone response curve, so as to expand their dynamic range. Ideally, this non-linearity would be removed, perhaps by estimating it during inference, or by measuring the curve from a series of bracketed 

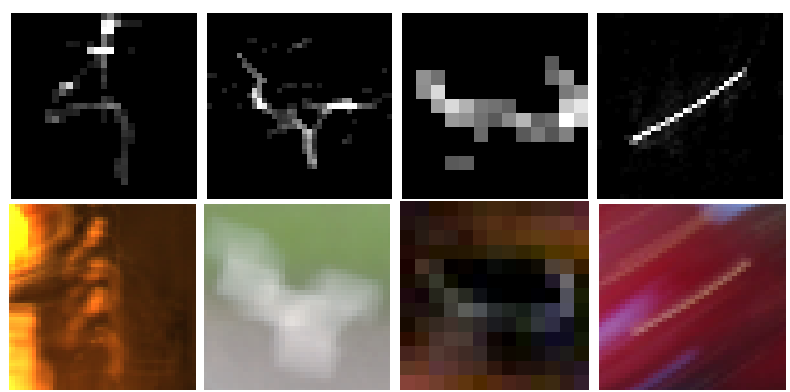

Figure 10: Top row: Inferred blur kernels from four real images (the cafe, fountain and family scenes plus another image not shown). Bottom row: Patches extracted from these scenes where the true kernel has been revealed. In the cafe image, two lights give a dual image of the kernel. In the fountain scene, a white square is transformed by the blur kernel. The final two images have specularities transformed by the camera motion, revealing the true kernel.
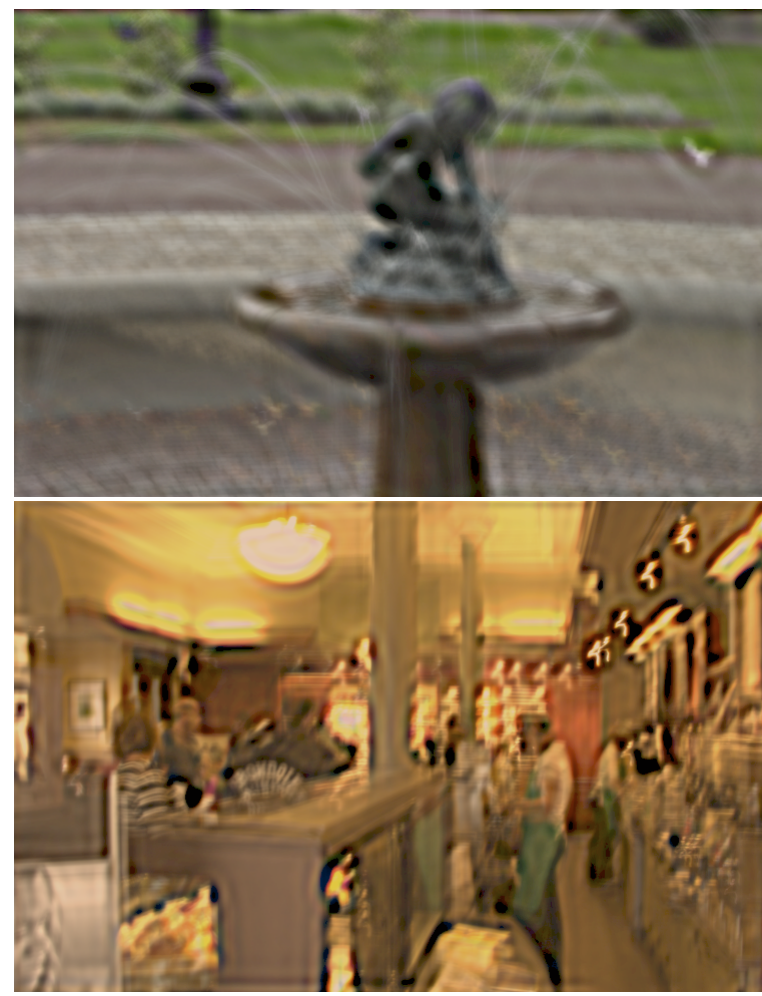

Figure 11: Baseline experiments, using Matlab's blind deconvolution algorithm deconvblind on the fountain image (top) and cafe image (bottom). The algorithm was initialized with a Gaussian blur kernel, similar in size to the blur artifacts.

exposures. Additionally, our method could be extended to make use of more advanced natural image statistics, such as correlations between color channels, or the fact that camera motion traces a continuous path (and thus arbitrary kernels are not possible). There is also room to improve the noise model in the algorithm; our current approach is based on Gaussian noise in image gradients, which is not a very good model for image sensor noise.

Although our method requires some manual intervention, we believe these steps could be eliminated by employing more exhaustive search procedures, or heuristics to guess the relevant parameters.
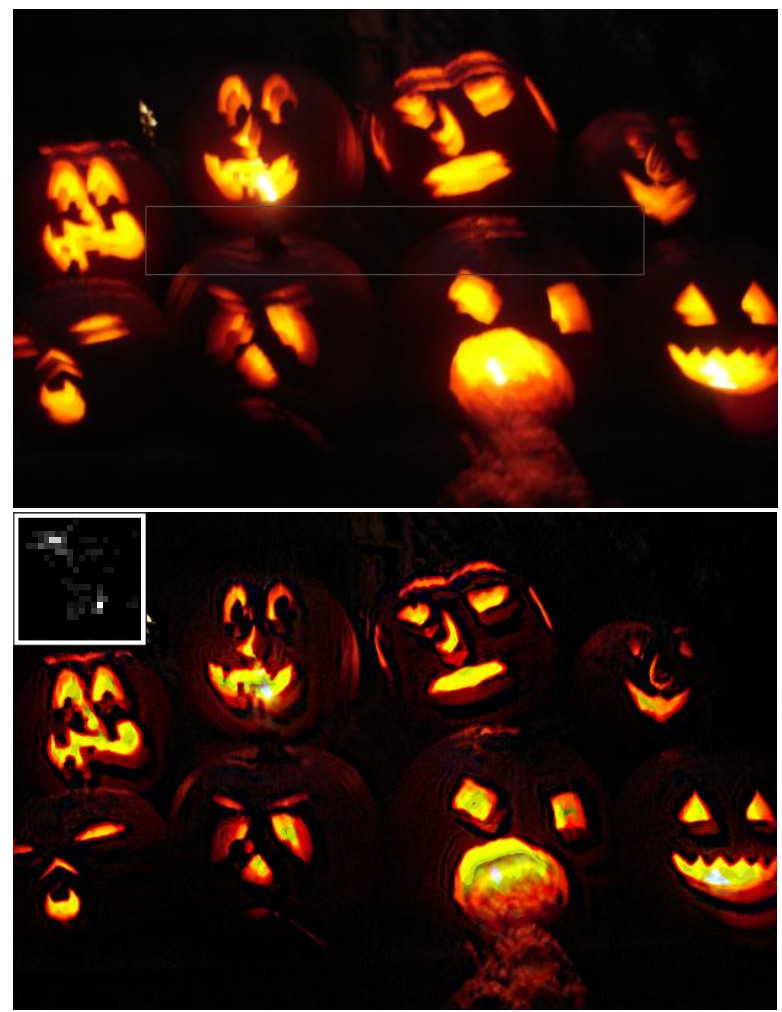

Figure 12: Top: A blurred scene with significant saturation. The long thin region selected by the user has limited saturation. Bottom: output of our algorithm. Note the double exposure type blur kernel.

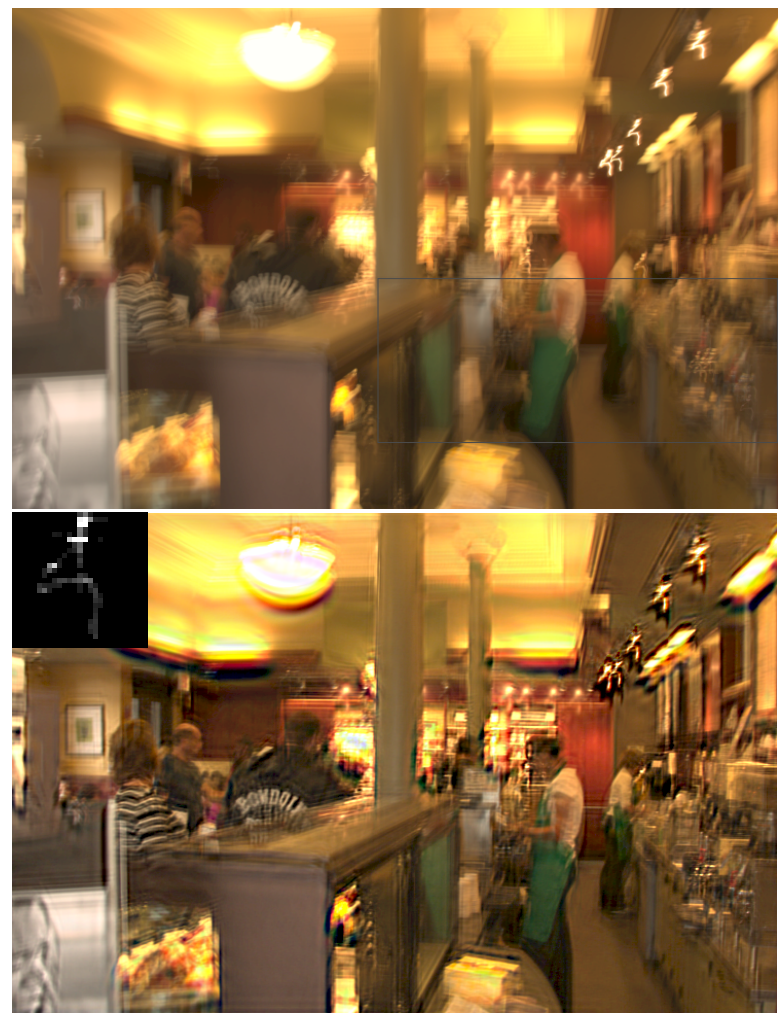

Figure 13: Top: A blurred scene with heavy saturation, taken with a 1 second exposure. Bottom: output of our algorithm. 


\section{Acknowledgements}

We are indebted to Antonio Torralba, Don Geman and Fredo Durand for their insights and suggestions. We are most grateful to James Miskin and David MacKay, for making their code available online. We would like the thank the following people for supplying us with blurred images for the paper: Omar Khan, Reinhard Klette, Michael Lewicki, Pietro Perona and Elizabeth Van Ruitenbeek. Funding for the project was provided by NSERC, NGA NEGI-1582-04-0004 and the Shell Group.

\section{References}

Apostoloff, N., AND FitzGibBon, A. 2005. Bayesian video matting using learnt image priors. In Conf. on Computer Vision and Pattern Recognition, 407-414.

Bascle, B., Blake, A., AND Zisserman, A. 1996. Motion Deblurring and Superresolution from an Image Sequence. In $E C C V(2), 573-582$

Ben-Ezra, M., And NAYAR, S. K. 2004. Motion-Based Motion Deblurring. IEEE Trans. on Pattern Analysis and Machine Intelligence 26, 6, 689-698.

BIGGS, D., AND ANDREws, M. 1997. Acceleration of iterative image restoration algorithms. Applied Optics 36, 8, 1766-1775.

CANON INC., 2006. What is optical image stabilizer? http://www.canon.com/ bctv/faq/optis.html.

CARON, J., NAMAZI, N., AND Rollins, C. 2002. Noniterative blind data restoration by use of an extracted filter function. Applied Optics 41, 32 (November), 68-84.

FIELD, D. 1994. What is the goal of sensory coding? Neural Computation 6, 559-601.

GEMAN, D., AND REYNOLDS, G. 1992. Constrained restoration and the recovery of discontinuities. IEEE Trans. on Pattern Analysis and Machine Intelligence 14, 3 , $367-383$.

GuLL, S. 1998. Bayesian inductive inference and maximum entropy. In Maximum Entropy and Bayesian Methods, J. Skilling, Ed. Kluwer, 54-71.

Jalobeanu, A., Blanc-Fraud, L., AND Zerubia, J. 2002. Estimation of blur and noise parameters in remote sensing. In Proc. of Int. Conf. on Acoustics, Speech and Signal Processing.

JANSSON, P. A. 1997. Deconvolution of Images and Spectra. Academic Press.

Jordan, M., Ghahramani, Z., JaAKKola, T., AND SaUl, L. 1999. An introduction to variational methods for graphical models. In Machine Learning, vol. 37, 183-233

Kundur, D., AND HatzinAKos, D. 1996. Blind image deconvolution. IEEE Signal Processing Magazine 13, 3 (May), 43-64.

LEVIn, A., AND WEISS, Y. 2004. User Assisted Separation of Reflections from a Single Image Using a Sparsity Prior. In ICCV, vol. 1, 602-613.

LEvin, A., ZOMET, A., AND WEISS, Y. 2003. Learning How to Inpaint from Global Image Statistics. In ICCV, 305-312.

LiU, X., AND Gamal, A. 2001. Simultaneous image formation and motion blur restoration via multiple capture. In Proc. Int. Conf. Acoustics, Speech, Signal Processing, vol. 3, 1841-1844.

LUCY, L. 1974. Bayesian-based iterative method of image restoration. Journal of Astronomy 79, 745-754.

Miskin, J., AND MACKAY, D. J. C. 2000. Ensemble Learning for Blind Image Separation and Deconvolution. In Adv. in Independent Component Analysis, M. Girolani, Ed. Springer-Verlag.

MisKin, J., 2000. Train ensemble library. http://www. inference.phy.cam.ac . $\mathrm{uk} / \mathrm{jwm} 1003 /$ train_ensemble.tar.gz.

MIskin, J. W. 2000. Ensemble Learning for Independent Component Analysis. PhD thesis, University of Cambridge.

Neelamani, R., Choi, H., AND Baraniuk, R. 2004. Forward: Fourier-wavelet regularized deconvolution for ill-conditioned systems. IEEE Trans. on Signal Processing 52 (Feburary), 418-433.

Rav-Acha, A., ANd Peleg, S. 2005. Two motion-blurred images are better than one. Pattern Recognition Letters, 311-317.

RICHARDSON, W. 1972. Bayesian-based iterative method of image restoration. Journal of the Optical Society of America A 62, 55-59.

Roth, S., AND BLACK, M. J. 2005. Fields of Experts: A Framework for Learning Image Priors. In $C V P R$, vol. 2, 860-867.

Simoncelli, E. P. 2005. Statistical modeling of photographic images. In Handbook of Image and Video Processing, A. Bovik, Ed. ch. 4.

TAPPEn, M. F., Russell, B. C., AND Freeman, W. T. 2003. Exploiting the sparse derivative prior for super-resolution and image demosaicing. In SCTV.

Tsumuraya, F., MiURA, N., AND BABA, N. 1994. Iterative blind deconvolution method using Lucy's algorithm. Astron. Astrophys. 282, 2 (Feb), 699-708.

WEISS, Y. 2001. Deriving intrinsic images from image sequences. In ICCV, 68-75.

ZAROWIN, C. 1994. Robust, noniterative, and computationally efficient modification of van Cittert deconvolution optical figuring. Journal of the Optical Society of America A 11, 10 (October), 2571-83.

\section{Appendix A}

Here we give pseudo code for the algorithm, Image Deblur. This calls the inference routine, Inference, adapted from Miskin and MacKay [2000a; 2000]. For brevity, only the key steps are detailed. Matlab notation is used. The Matlab functions imresize, edgetaper and deconvlucy are used with their standard syntax.
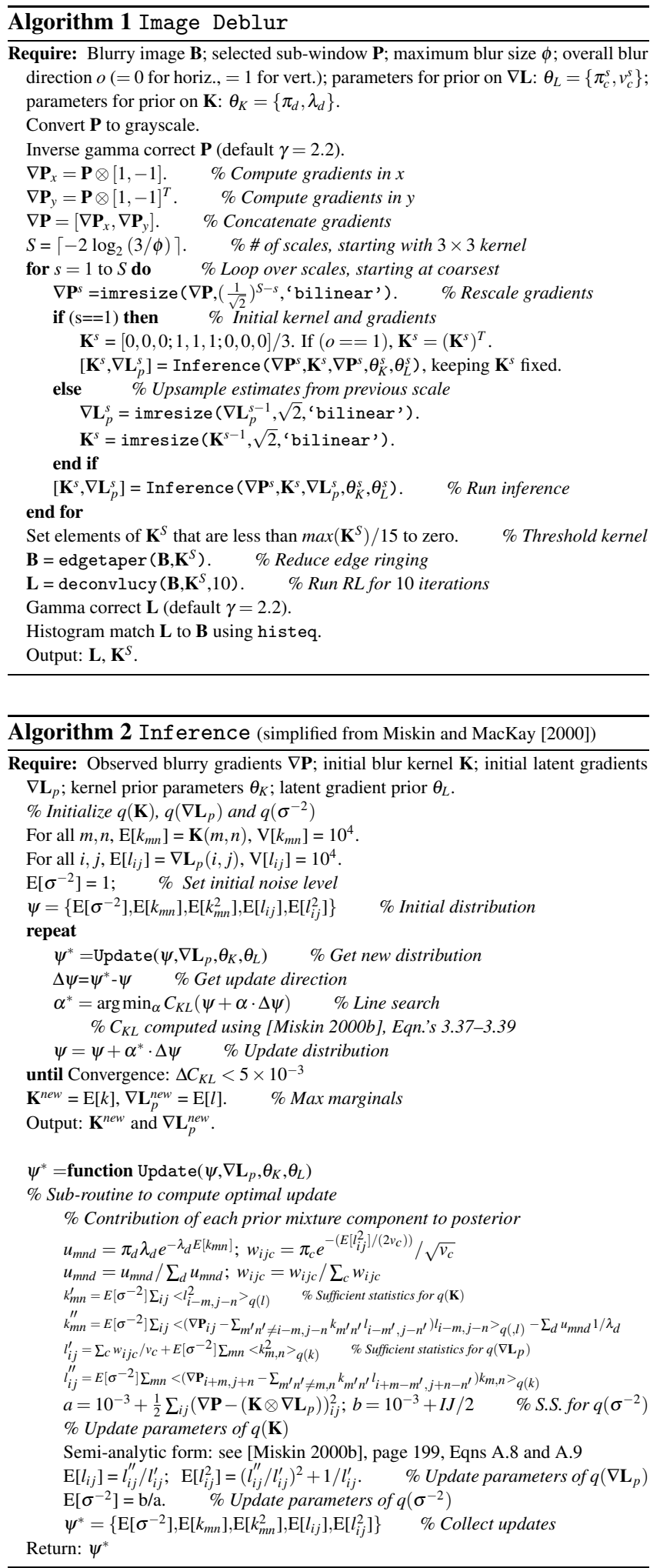